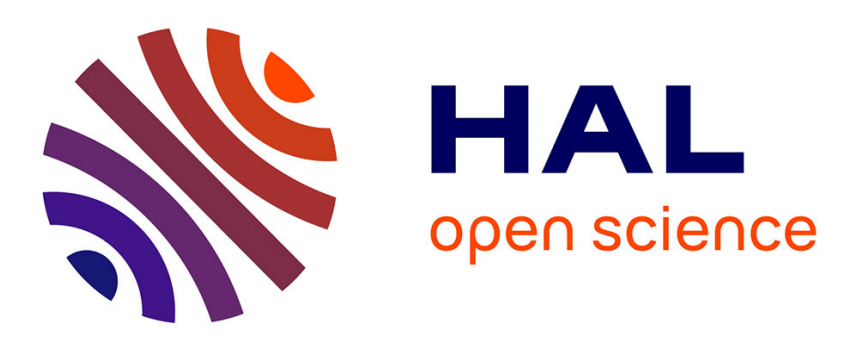

\title{
Strength of a matrix with elliptic criterion reinforced by rigid inclusions with imperfect interfaces
}

François Bignonnet, Luc Dormieux, Éric Lemarchand

\section{To cite this version:}

François Bignonnet, Luc Dormieux, Éric Lemarchand. Strength of a matrix with elliptic criterion reinforced by rigid inclusions with imperfect interfaces. European Journal of Mechanics - A/Solids, 2015, 52, pp.95-106. 10.1016/j.euromechsol.2015.02.003 . hal-01337841

\section{HAL Id: hal-01337841 \\ https://hal.science/hal-01337841}

Submitted on 27 Jun 2016

HAL is a multi-disciplinary open access archive for the deposit and dissemination of scientific research documents, whether they are published or not. The documents may come from teaching and research institutions in France or abroad, or from public or private research centers.
L'archive ouverte pluridisciplinaire HAL, est destinée au dépôt et à la diffusion de documents scientifiques de niveau recherche, publiés ou non, émanant des établissements d'enseignement et de recherche français ou étrangers, des laboratoires publics ou privés. 


\title{
Strength of a matrix with elliptic criterion reinforced by rigid inclusions with imperfect interfaces
}

\author{
François Bignonnet*, Luc Dormieux, Eric Lemarchand \\ Université Paris-Est, Laboratoire Navier (UMR 8205), CNRS, ENPC, IFSTTAR, F-77455 Marne-la-Vallée, France
}

\begin{abstract}
Elliptic effective strength criteria in the mean-deviatoric stress plane are encountered in porous media for a granular material made of rigid grains with cohesive frictional interfaces or a material with pores in a Drucker-Prager matrix. The macroscopic strength criterion of a heterogeneous material comprising a matrix with elliptic strength criterion reinforced by rigid inclusions with perfect or imperfect interfaces is studied. Considered imperfect interfaces follow either a Tresca or a Mohr-Coulomb strength criterion. Derived macroscopic criteria are shown to be a combination of a larger ellipse, which corresponds to the criterion for perfectly bounded interfaces, conditionally truncated by a smaller ellipse resulting from the activation of interfacial mechanisms. The activation of the interfacial mechanisms depends on the matrix and interfaces strength properties, inclusions concentration, as well as the macroscopic strain triaxiality ratio.
\end{abstract}

Keywords: Strength, Homogenization, Interfaces, Micromechanics, Mohr-Coulomb, Tresca

\section{Introduction}

Imperfect interfaces between constituents of heterogeneous media are increasingly understood to play a major role on the effective strength properties. Such imperfect interfaces can be characterized by a criterion the stress vector acting on the interface must not exceed. Thanks to homogenization methods, the strength of granular geomaterials has been investigated, successively considering the cases of rigid grains interfaced by a Tresca criterion (Dormieux et al., 2007), a frictional criterion (Maalej et al., 2009) or a cohesive frictional criterion (He et al., 2013), as well as the competition between Tresca interfacial and Von Mises intra-granular strength (Dormieux et al., 2010).

Additionally, several types of porous media have been recognized to be governed by an elliptic effective strength criterion in the mean-deviatoric stress plane. For example, a composite made of pores in a matrix with a Von Mises (Barthélémy, 2005) or Drucker-Prager (Barthélémy, 2005; Maghous et al., 2009) strength criterion has an elliptic macroscopic strength criterion. Above a critical porosity threshold, the granular material with cohesive frictional interfaces considered in He et al. (2013) also proves to follow a similar criterion. Elliptic strength criteria are thus of great interest in geomechanics, for instance to describe the clay matrix of a shale. However, it is worth noting that this class of elliptic strength criteria is obtained only by applying the so-called modified secant modulii approach (Suquet, 1995) to the homogenization of ductile porous materials, which is precisely the method we intend to adopt in the present work for a second homogenization step. A Gurson-type analysis (Gurson, 1977) of the strength of porous materials would have led to the use of a different criterion for the clay matrix, but will not be considered here.

\footnotetext{
*Corresponding author. Tel.: +33 164153750

Email addresses: francois.bignonnet@enpc.fr (François Bignonnet), luc.dormieux@enpc.fr (Luc Dormieux), eric.lemarchand@enpc.fr (Eric Lemarchand)
} 


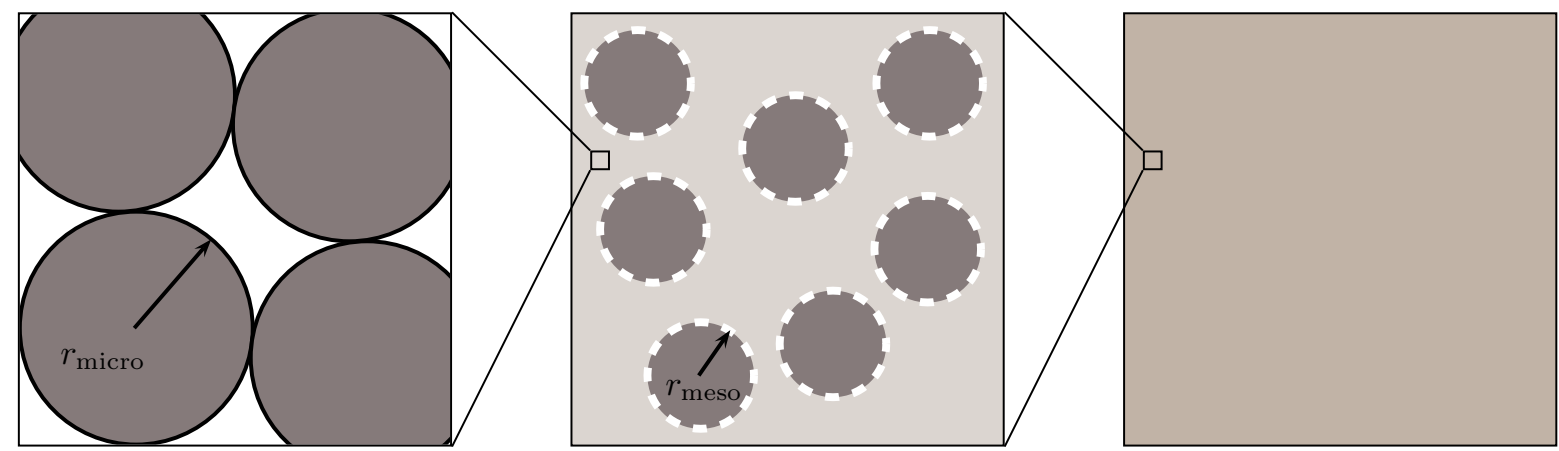

rigid grains

rigid inclusions

Figure 1: Example of thought-of material. Cohesive frictional granular material reinforced by rigid particles with imperfect interfaces. According to the separation of scale principle, $r_{\text {micro }} \ll r_{\text {meso }}$. This paper deals with upscaling from meso to macro scales.

Driven by the thought-of example of shales for which the clay matrix is reinforced by silica or calcite inclusions (Fig. 1), the macroscopic strength of a material made of a matrix with an elliptic strength criterion reinforced by rigid inclusions is yet to be investigated. Furthermore, the degradation of this reinforcement, coming from matrix-inclusion interface imperfections, is of critical interest. The case of perfect interfaces will be compared to Tresca or Mohr-Coulomb interfaces. The present work addresses the imperfection of the interfaces under the hypothesis of ductility, whereas matrix-inclusion debonding had previously been studied in the context of fracture mechanics (see e.g. Mantič (2009) and Greco et al. (2013)).

This paper aims at addressing this strength issue using continuum micromechanics. From a technical point of view, Barthélémy and Dormieux (2004) developed a strength homogenization method in which the macroscopic stress states lying on the boundary of the effective strength criterion domain are obtained by solving a fictitious non linear viscous problem. In turn, the non linear problem can be solved using secant or affine methods (Suquet, 1995, 1997) which rely on the solution to the associated linear problem. These methods proved successful to predict the strength of heterogeneous material, even in the presence of interface effects (Barthélémy, 2005; Barthélémy and Dormieux, 2004; Dormieux et al., 2010, 2006, 2007; He et al., 2013; Maalej et al., 2009; Maghous et al., 2009; Sanahuja and Dormieux, 2005).

To start with, the homogenization method is briefly recalled and the fictitious non linear problem is derived from the strength properties of the components in section 2 .

Next, the non linear homogenization method is presented in section 3 after resolution of the linear problem underlying the fictitious problem arising from the previous section.

Finally, the macroscopic strength criteria are derived in section 4 in the case of perfect, Tresca or MohrCoulomb interfaces.

Notations The second and fourth order identity tensors are respectively denoted by $\mathbf{1}$ and $\mathbb{I}$. The volumic and deviatoric projection tensors $\mathbb{J}$ and $\mathbb{K}$ are defined as $\mathbb{J}=\frac{1}{3} \mathbf{1} \otimes \mathbf{1}$ and $\mathbb{K}=\mathbb{I}-\mathbb{J}$.

\section{Limit state equations}

\subsection{Methodology}

The aim of this article is to determine the effective strength of a composite made of a matrix reinforced by rigid inclusions with imperfect matrix-inclusion interfaces. A representative elementary volume $(r e v) \Omega$ of this composite is introduced. It comprises a matrix (phase $\Omega_{m}$ ) and rigid inclusions (phase $\Omega_{i}$ ) with 
volume fraction $\rho$. Locally, the unit normal to the interface directed outwards from the inclusion is noted $\boldsymbol{n}$. The volume averages of a function a over the rev $\Omega$, the matrix phase $\Omega_{m}$, the inclusionary phase $\Omega_{i}$, and all the interfaces $\Gamma$ are respectively denoted $\bar{a}^{\Omega}=\bar{a}, \bar{a}^{m}, \bar{a}^{i}$ and $\bar{a}^{\Gamma}$.

A macroscopic stress state $\Sigma$ applied to $\Omega$ is said to be admissible provided that there exists some microscopic stress field $\sigma(\boldsymbol{z})$ defined on the rev $\Omega$ which meets the following conditions (de Buhan (1986), Suquet (1983)):

$$
\begin{array}{ll}
\operatorname{div} \boldsymbol{\sigma}=0 & (\forall \boldsymbol{z} \in \Omega) \\
\boldsymbol{\sigma}(\boldsymbol{z}) \in G(\boldsymbol{z}) & (\forall \boldsymbol{z} \in \Omega) \\
\overline{\boldsymbol{\sigma}}=\Sigma &
\end{array}
$$

where $G(\boldsymbol{z})$ denotes the domain of admissible microscopic stress states at point $\boldsymbol{z}$ in the rev $\Omega$. The set of admissible macroscopic stress states is denoted by $G^{\text {hom }}$.

The strength properties of the constituents at the microscopic scale therefore need to be characterized. The domain $G_{m}$ of admissible stress states in the matrix is defined by a strength criterion $f_{m}(\boldsymbol{\sigma})$ such that:

$$
\boldsymbol{\sigma} \in G_{m} \Leftrightarrow f_{m}(\boldsymbol{\sigma}) \leqslant 0
$$

In turn, the inclusions are supposed infinitely resistant. The emphasis of this paper is put on the limited strength of the matrix-inclusions interfaces: The inclusions may be not perfectly bounded to the matrix; instead, the strength of the interface is described by a criterion on the stress vector $\boldsymbol{T}$ acting on the matrixinclusion interface:

$$
\boldsymbol{T} \in G_{\Gamma} \Leftrightarrow f_{\Gamma}(\boldsymbol{T}) \leqslant 0 .
$$

$\Gamma$ denotes the set of matrix-inclusion interfaces and $G_{\Gamma}$ defined above is the set of admissible stress vectors.

Equivalently, the domains $G_{m}$ and $G_{\Gamma}$ may be characterized by their support functions (Dormieux et al., 2006; Salencon, 1990). This is the so-called dual formulation. The support function $\pi_{m}(\boldsymbol{d})$ of the matrix strength is defined as

$$
\pi_{m}(\boldsymbol{d})=\sup \left\{\boldsymbol{\sigma}: \boldsymbol{d}, f_{m}(\boldsymbol{\sigma}) \leqslant 0\right\},
$$

where $\boldsymbol{d}$ and $\pi_{m}(\boldsymbol{d})$ physically represent a virtual strain rate and the associated dissipation. Likewise, the support function $\pi_{\Gamma}(\llbracket \boldsymbol{v} \rrbracket)$ of the interface criterion is

$$
\pi_{\Gamma}(\llbracket \boldsymbol{v} \rrbracket)=\sup \left\{\boldsymbol{T}: \llbracket \boldsymbol{v} \rrbracket, f_{\Gamma}(\boldsymbol{T}) \leqslant 0\right\},
$$

where $\llbracket \boldsymbol{v} \rrbracket$ and $\pi_{\Gamma}(\llbracket \boldsymbol{v} \rrbracket)$ physically represent a virtual velocity jump across the interface and the associated dissipation.

The direct use of the definition (1) for the determination of $G^{\text {hom }}$ is uneasy. Alternatively, as shown in Leblond et al. (1994),Barthélémy and Dormieux (2004), the boundary $\partial G^{\text {hom }}$ of the macroscopic strength criterion can be retrieved by solving the following boundary value problem defined on the rev $\Omega$

$$
\begin{array}{ll}
\operatorname{div} \boldsymbol{\sigma}=0 & (\Omega) \\
\boldsymbol{\sigma}=\frac{\partial \pi_{m}}{\partial \boldsymbol{d}} & \left(\Omega_{m}\right) \\
\boldsymbol{T}=\frac{\partial \pi_{\Gamma}}{\partial \llbracket \boldsymbol{v} \rrbracket} & (\Gamma) \\
\boldsymbol{\sigma}=\mathbb{C}_{i}: \boldsymbol{d} & \left(\Omega_{i}\right) \text { with } \mathbb{C}_{i} \rightarrow \infty \\
\boldsymbol{v}(\boldsymbol{z})=\boldsymbol{D} \cdot \boldsymbol{z} & (\partial \Omega) \\
\boldsymbol{d}=\operatorname{grad}^{s} \boldsymbol{v} & (\Omega)
\end{array}
$$


This boundary value problem may be interpreted as a fictitious non linear viscous problem where $\boldsymbol{\sigma}$ is the local stress field and $\boldsymbol{v}$ the local velocity field. The important feature is that the macroscopic stress state $\boldsymbol{\Sigma}$, related to $\boldsymbol{\sigma}$ by the stress averaging rule $\boldsymbol{\Sigma}=\overline{\boldsymbol{\sigma}}$ lies on the boundary $\partial G^{\text {hom }}$ of the macroscopic strength criterion. More precisely it is located at the point of $\partial G^{\text {hom }}$ where the outward normal is oriented along the macroscopic strain rate $\boldsymbol{D}$.

Thus, the determination of the boundary $\partial G^{\text {hom }}$ of the macroscopic strength criterion amounts to solving the above fictitious non linear viscous problem. The non linear viscous state equations in each phase are defined from the derivatives of their support function. The next parts are dedicated to the characterization of the strength properties of the matrix and the interfaces and to the derivation of the associated fictitious state equations.

\subsection{Matrix}

Using appropriate non linear homogenization techniques, He et al. (2013) recently derived the macroscopic strength of a granular medium made of rigid spherical grains interfaced by cohesive frictional interfaces. The latter follow a Mohr-Coulomb failure criterion. It will be assumed that the result of this analysis provides a suitable description for the strength of the matrix at stake. The strength criterion is isotropic and can be expressed as follows:

$$
f_{m}(\boldsymbol{\sigma})=\frac{\left(\sigma_{m}+c\right)^{2}}{a}+\frac{\sigma_{d}^{2}}{b}-1 \leqslant 0
$$

where a, b, c depend on intergranular interfacial strength properties and on the porosity. The stress invariant appearing in (3) are the mean stress $\sigma_{m}$ and the deviatoric stress $\sigma_{d}$, which are defined as

$$
\sigma_{m}=\frac{1}{3} \operatorname{tr}(\boldsymbol{\sigma}) \quad ; \quad \boldsymbol{\sigma}_{d}=\boldsymbol{\sigma}-\sigma_{m} \mathbf{1} \quad ; \quad \sigma_{d}=\sqrt{\boldsymbol{\sigma}_{d}: \boldsymbol{\sigma}_{d}}
$$

In the $\left(\sigma_{m}, \sigma_{d}\right)$ plane, the boundary of $G_{m}$ is an ellipse with semi-axes $\sqrt{a}$ and $\sqrt{b}$. The support function of the failure criterion (3) is the function of the tensorial variable $\boldsymbol{d}$

$$
\pi_{m}(\boldsymbol{d})=\sqrt{a d_{v}^{2}+b d_{d}^{2}}-c d_{v}
$$

where the strain rate invariants are the volume strain rate $d_{v}$ and the deviatoric strain rate $d_{d}$, defined as

$$
d_{v}=\operatorname{tr}(\boldsymbol{d}) \quad ; \quad \boldsymbol{d}_{d}=\boldsymbol{d}-\frac{1}{3} d_{v} \mathbf{1} \quad ; \quad d_{d}=\sqrt{\boldsymbol{d}_{d}: \boldsymbol{d}_{d}}
$$

Differentiating the support function (4), the fictitious state equation in the matrix is written in a secant formulation involving a prestress

$$
\boldsymbol{\sigma}=\frac{\partial \pi_{m}}{\partial \boldsymbol{d}}=\mathbb{C}_{m}: \boldsymbol{d}+\boldsymbol{\sigma}^{p}
$$

with

$$
\begin{aligned}
& \mathbb{C}_{m}=3 k_{m} \mathbb{J}+2 \mu_{m} \mathbb{K} \quad ; \quad \boldsymbol{\sigma}^{p}=-c \mathbf{1} \\
& k_{m}=\frac{a}{\sqrt{a d_{v}^{2}+b d_{d}^{2}}} \quad ; \quad 2 \mu_{m}=\frac{b}{\sqrt{a d_{v}^{2}+b d_{d}^{2}}} .
\end{aligned}
$$

\subsection{Tresca interfaces}

First, the case where the strength of the interfaces is governed by a Tresca criterion $f_{\operatorname{tr}}(\boldsymbol{T})$ is considered. The Tresca criterion is characterized by a threshold $k$ on the tangential component $T_{t}$ of the stress vector:

$$
f_{\mathrm{tr}}(\boldsymbol{T})=T_{t}-k \leqslant 0
$$


with

$$
\boldsymbol{T}_{t}=(\mathbf{1}-\boldsymbol{n} \otimes \boldsymbol{n}) \cdot \boldsymbol{T} \quad ; \quad T_{t}=\sqrt{\boldsymbol{T}_{t} \cdot \boldsymbol{T}_{t}} \quad ; \quad T_{n}=\boldsymbol{T} \cdot \boldsymbol{n} .
$$

The support function of the Tresca criterion (Salencon, 1990) is

$$
\pi_{\operatorname{tr}}(\llbracket \boldsymbol{v} \rrbracket)= \begin{cases}+\infty & \text { if } \llbracket v_{n} \rrbracket \neq 0 \\ k \llbracket v_{t} \rrbracket & \text { if } \llbracket v_{n} \rrbracket=0 .\end{cases}
$$

This support function is highly singular, thus not suited for the direct implementation from the definition of the fictitious state equation. Following Barthélémy (2005); Sanahuja and Dormieux (2005), the Tresca criterion can be viewed as the limit of a sequence of ellipses in the $\left(T_{n}, T_{t}\right)$ plan

$$
f_{\mathrm{tr}}(\boldsymbol{T}, L)=\left(\frac{T_{n}}{L}\right)^{2}+\left(\frac{T_{t}}{k}\right)^{2}-1 \leqslant 0,
$$

when $L / k$ tends to infinity. The support function of the regularized criterion (8) is

$$
\pi_{\mathrm{tr}}(\llbracket \boldsymbol{v} \rrbracket, L)=\sqrt{\left(L \llbracket v_{n} \rrbracket\right)^{2}+\left(k \llbracket v_{t} \rrbracket\right)^{2}} .
$$

The associated state equation reads

$$
\boldsymbol{T}=\frac{\partial \pi_{\operatorname{tr}}(\llbracket \boldsymbol{v} \rrbracket, L)}{\partial \llbracket \boldsymbol{v} \rrbracket}=\boldsymbol{K}(\llbracket \boldsymbol{v} \rrbracket, L) \cdot \llbracket \boldsymbol{v} \rrbracket=K_{n}(\llbracket \boldsymbol{v} \rrbracket, L) \llbracket v_{n} \rrbracket \boldsymbol{n}+K_{t}(\llbracket \boldsymbol{v} \rrbracket, L) \llbracket \boldsymbol{v}_{t} \rrbracket,
$$

where the normal and tangential components of the velocity jump $\llbracket \boldsymbol{v} \rrbracket$ across the interface are defined similarly to those of the stress vector $\boldsymbol{T}$. The secant modulii appearing in (10) are:

$$
K_{n}(\llbracket \boldsymbol{v} \rrbracket, L)=\frac{L^{2}}{\sqrt{\left(L \llbracket v_{n} \rrbracket\right)^{2}+\left(k \llbracket v_{t} \rrbracket\right)^{2}}} \quad ; \quad K_{t}(\llbracket \boldsymbol{v} \rrbracket, L)=\frac{k^{2}}{\sqrt{\left(L \llbracket v_{n} \rrbracket\right)^{2}+\left(k \llbracket v_{t} \rrbracket\right)^{2}}} .
$$

In the limit $L / k \rightarrow \infty$, the normal velocity jump $\llbracket v_{n} \rrbracket \rightarrow 0$ while tangential velocity jumps are allowed. Then, the tangent stiffness modulus $K_{t} \rightarrow k / \llbracket v_{t} \rrbracket$.

\subsection{Mohr-Coulomb interfaces}

In order to account for the effect of the normal stress on the strength of a frictional interface, let now assume that the stress vector at the matrix-inclusion interface is bounded by a Mohr-Coulomb criterion:

$$
f_{\mathrm{mc}}(\boldsymbol{T})=T_{t}+\alpha\left(T_{n}-h\right) \leqslant 0 .
$$

The positive scalars $h$ and $\alpha$ respectively denote the interface tensile strength and the friction coefficient. The support function of the Mohr-Coulomb criterion (Salencon, 1990) is

$$
\pi_{\mathrm{mc}}(\llbracket \boldsymbol{v} \rrbracket)= \begin{cases}+\infty & \text { if } \llbracket v_{n} \rrbracket<\alpha \llbracket v_{t} \rrbracket \\ h \llbracket v_{n} \rrbracket & \text { if } \llbracket v_{n} \rrbracket \geqslant \alpha \llbracket v_{t} \rrbracket .\end{cases}
$$

The support function is highly singular. Again, in view of deriving the fictitious state equation of this interface, a direct differentiation is not possible. Hence, following He et al. (2013); Maghous et al. (2009), the support function has to be regularized by a sequence of potentials

$$
\psi_{j}(\llbracket \boldsymbol{v} \rrbracket)=f_{j}(Y)+h \llbracket v_{n} \rrbracket \text { with } Y=\llbracket v_{n} \rrbracket-\alpha \llbracket v_{t} \rrbracket .
$$

The positive scalar $j$ will tend to 0 and $f_{j}$ is a convex function of class $C^{2}$ defined on ] $-j,+\infty[$ by

- $f_{j}(Y)=0$ if $Y \geqslant 0$, 
- $f_{j}$ decreasing on $\left.]-j, 0\right]$,

- $\lim _{Y \rightarrow-j^{+}} f_{j}(Y)=+\infty$.

Thus, when $j$ tends to 0 , the sequence of potentials $\psi_{j}$ tends to the support function $\pi_{\mathrm{mc}}$ of the interface criterion. The state equation associated with the regularized potential is

$$
\boldsymbol{T}=\frac{\partial \psi_{j}}{\partial \llbracket \boldsymbol{v} \rrbracket}=\frac{\partial \psi_{j}}{\partial \llbracket v_{n} \rrbracket} \boldsymbol{n}+\frac{\partial \psi_{j}}{\partial \llbracket v_{t} \rrbracket} \boldsymbol{t}
$$

Introducing the function $\mathcal{F}(Y)=\frac{\partial \psi_{j}}{\partial Y}$ which is negative or null, the state equation is

$$
\boldsymbol{T}=\mathcal{F}(Y)(\boldsymbol{n}-\alpha \boldsymbol{t})+h \boldsymbol{n} .
$$

Hence, for $\boldsymbol{T} \neq h \boldsymbol{n}$, necessarily $\mathcal{F}(Y)<0$ and thus $Y \in]-j ; 0\left[\right.$. Asymptotically, the flow rule $\llbracket v_{n} \rrbracket=\alpha \llbracket v_{t} \rrbracket$ is met as $j$ tends to 0 . This feature of the described technique is very important in so far as it means that the normality rule in the interfaces is correctly accounted for.

In order to have positive tangential and normal modulii, the state equation is written in an affine form:

$$
\boldsymbol{T}=\boldsymbol{K}\left(\llbracket v_{n} \rrbracket, \llbracket v_{t} \rrbracket\right) \cdot \llbracket \boldsymbol{v} \rrbracket+\boldsymbol{T}^{p}\left(\llbracket v_{n} \rrbracket, \llbracket v_{t} \rrbracket\right) .
$$

where

$$
K_{n}=\frac{\mathcal{F}}{Y} \quad ; \quad K_{t}=-\frac{\alpha \mathcal{F}}{\llbracket v_{t} \rrbracket} \quad ; \quad \boldsymbol{T}^{p}=\left(h-\frac{\alpha \mathcal{F}}{Y} \llbracket v_{t} \rrbracket\right) \boldsymbol{n} .
$$

Asymptotically, the affine modulii meet

$$
\lim _{j \rightarrow 0} \frac{K_{t}}{K_{n}}=\lim _{j \rightarrow 0}-\frac{\alpha Y}{v_{t}}=0 \text { and } \lim _{j \rightarrow 0} \frac{T^{p}}{K_{n}}=-\alpha v_{t} .
$$

\section{Linear and non linear homogenization}

\subsection{Linear homogenization}

As stated before, the dual approach for limit analysis developed in the previous section leads to a fictitious non linear viscous problem. As far as homogenization is concerned, the modified secant modulii method (see e.g. Suquet $(1995,1997)$ ) provides an efficient technique to deal with non linearity. It relies upon the solution of a linear homogenization problem together with the derivation of appropriate phase average quantities. For this reason, this section is devoted to the resolution of the linear viscous problem and to the derivation of average estimates for the strain rate in the matrix as well as for the velocity jump in interfaces.

\subsubsection{Definition of the linear problem}

To account for the prestressed formulation of the matrix and interface state equations, the linear problem must include three loading parameters: the macroscopic strain rate $\boldsymbol{D}$, the matrix uniform prestress $p$ and the interface uniform prestress $\varpi$. The linear problem is thus

$$
\begin{array}{ll}
\operatorname{div} \boldsymbol{\sigma}=0 & (\Omega) \\
\boldsymbol{\sigma}=\mathbb{C}_{m}: \boldsymbol{d}-p \mathbf{1} & \left(\Omega_{m}\right) \\
\boldsymbol{T}=\boldsymbol{K} \cdot \llbracket \boldsymbol{v} \rrbracket+\varpi \boldsymbol{n} & (\Gamma) \\
\boldsymbol{\sigma}=\mathbb{C}_{i}: \boldsymbol{d} & \left(\Omega_{i}\right) \text { with } \mathbb{C}_{i} \rightarrow \infty \\
\boldsymbol{v}(\boldsymbol{z})=\boldsymbol{D} \cdot \boldsymbol{z} & (\partial \Omega) \\
\boldsymbol{d}=\operatorname{grad}^{s} \boldsymbol{v} & (\Omega)
\end{array}
$$

The linear problem (20) is solved by superposition of the following loading modes 
- (P1) : no prestress, macroscopic strain $\boldsymbol{D}$ at the boundary $\partial \Omega$.

- $(\mathrm{P} 2)$ : prestress $-(p+\varpi) \mathbf{1}$ in the matrix only, no prestress in the interfaces, macroscopic strain $\mathbf{0}$ at the boundary $\partial \Omega$.

- (P3) : prestress $\varpi \mathbf{1}$ in the matrix and $\varpi \boldsymbol{n}$ in the interfaces, macroscopic strain $\mathbf{0}$ at the boundary $\partial \Omega$

The local solution of problem ( $\mathrm{P} i)$ for $i=1,2,3$ is denoted $\left(\boldsymbol{v}_{i}, \boldsymbol{\sigma}_{i}\right)$, the associated strain rate is $\boldsymbol{d}_{i}$ and the macroscopic stress is $\boldsymbol{\Sigma}_{i}$. The solution of (P3) is the uniform couple $\boldsymbol{\sigma}_{3}=\varpi \mathbf{1}$ and $\boldsymbol{v}_{3}=\mathbf{0}$. The resolution of (P1) will yield the homogenized stiffness $\mathbb{C}^{\text {hom }}$ such that $\boldsymbol{\Sigma}_{1}=\mathbb{C}^{\text {hom }}: \boldsymbol{D}$.

The macroscopic potential energy density $\Psi$ of the linear problem (20) comprises the terms of elastic energy and the work of the prestresses

$$
\begin{aligned}
|\Omega| \Psi(\boldsymbol{D}, p, \varpi) & =\frac{1}{2} \int_{\Omega_{m}} \boldsymbol{d}: \mathbb{C}: \boldsymbol{d} \mathrm{d} V+\frac{1}{2} \int_{\Gamma} \llbracket \boldsymbol{v} \rrbracket: K: \llbracket \boldsymbol{v} \rrbracket \mathrm{d} S \\
& -(p+\varpi) \int_{\Omega_{m}} \operatorname{tr}(\boldsymbol{d}) \mathrm{d} V+\varpi \int_{\Omega} \operatorname{tr}(\boldsymbol{d}) \mathrm{d} V .
\end{aligned}
$$

Akin to poroelasticity where the change in pore volume fraction is the dual macroscopic state variable associated to pore pressure, let's introduce here an additional macroscopic state variable defined as the dual quantity of the matrix prestress. This dual quantity is the matrix volume change normalized by the initial volume of the rev:

$$
v_{i_{m}}=(1-\rho) \operatorname{tr}\left({\overline{\boldsymbol{d}_{i}}}^{m}\right) \quad(i=1,2)
$$

and by superposition:

$$
v_{m}=(1-\rho) \operatorname{tr}\left(\overline{\boldsymbol{d}}^{m}\right)
$$

Using the superposition rules $\boldsymbol{v}=\boldsymbol{v}_{1}+\boldsymbol{v}_{2}$ and $\boldsymbol{d}=\boldsymbol{d}_{1}+\boldsymbol{d}_{2}$, the density of potential energy $\Psi(\boldsymbol{D}, p, \varpi)$ reads

$$
\begin{aligned}
|\Omega| \Psi(\boldsymbol{D}, p, \varpi) & =\frac{1}{2} \int_{\Omega_{m}} \boldsymbol{d}_{1}: \mathbb{C}: \boldsymbol{d}_{1} \mathrm{~d} V+\frac{1}{2} \int_{\Gamma} \llbracket \boldsymbol{v}_{1} \rrbracket \cdot \boldsymbol{K} \cdot \llbracket \boldsymbol{v}_{1} \rrbracket \mathrm{d} S \\
& +\frac{1}{2} \int_{\Omega_{m}} \boldsymbol{d}_{2}:\left(\boldsymbol{\sigma}_{2}+(p+\varpi) \mathbf{1}\right) \mathrm{d} V+\frac{1}{2} \int_{\Gamma} \llbracket \boldsymbol{v}_{2} \rrbracket \cdot\left(\boldsymbol{\sigma}_{2} \cdot \boldsymbol{n}\right) \mathrm{d} S \\
& +\int_{\Omega_{m}} \boldsymbol{d}_{2}: \boldsymbol{\sigma}_{1} \mathrm{~d} V+\int_{\Gamma} \llbracket \boldsymbol{v}_{2} \rrbracket \cdot\left(\boldsymbol{\sigma}_{1} \cdot \boldsymbol{n}\right) \mathrm{d} S \\
& +|\Omega|\left(-(p+\varpi) v_{m}+\varpi \operatorname{tr} \boldsymbol{D}\right) .
\end{aligned}
$$

According to the energy definition of $\mathbb{C}^{\text {hom }}$, the sum of the two first integrals of the rhs is equal to

$$
\frac{|\Omega|}{2} \boldsymbol{D}: \mathbb{C}^{\text {hom }}: \boldsymbol{D} .
$$

The second and the third line can be simplified by application of Hill's lemma extended to interfaces (Maalej et al., 2009) to the couples of kinematically (resp. statically) admissible velocity and stress fields $\left(\boldsymbol{v}_{2}, \boldsymbol{\sigma}_{2}\right)$ and $\left(\boldsymbol{v}_{2}, \boldsymbol{\sigma}_{1}\right)$. Eventually, one obtains:

$$
\Psi(\boldsymbol{D}, p, \varpi)=\frac{1}{2} \boldsymbol{D}: \mathbb{C}^{\text {hom }}: \boldsymbol{D}-(p+\varpi)\left(v_{1_{m}}+\frac{1}{2} v_{2_{m}}\right)+\varpi \operatorname{tr} \boldsymbol{D} .
$$




\subsubsection{Quadratic effective estimates}

In view of the application of the modified secant modulii method to solve the non linear problem, volume and deviatoric estimates of the strain rate in the matrix are needed. In the modified secant modulii method, these effective values are defined as the quadratic averages over the matrix phase

$$
d_{v}^{\mathrm{eff}}=\sqrt{{\overline{\bar{d}_{v}^{2}}}^{m}} \quad ; \quad d_{d}^{\mathrm{eff}}=\sqrt{{\overline{d_{d}^{2}}}^{m}} .
$$

Following Dormieux et al. (2002); He et al. (2013), adapted from Kreher (1990), the estimates are

$$
\begin{aligned}
& \frac{1}{2}(1-\rho)\left(d_{v}^{\mathrm{e} f f}\right)^{2}=\frac{\partial \Psi(\boldsymbol{D}, p, \varpi)}{\partial k_{m}}, \\
& \frac{1}{2}(1-\rho)\left(d_{d}^{\mathrm{e} f f}\right)^{2}=\frac{\partial \Psi(\boldsymbol{D}, p, \varpi)}{\partial 2 \mu_{m}} .
\end{aligned}
$$

Similarly, effective jump of velocity at the interface is defined as

$$
\llbracket v_{n} \rrbracket^{\mathrm{eff}}=\sqrt{{\overline{\llbracket v_{n} \rrbracket^{2}}}^{\Gamma}} ; \quad \llbracket v_{t} \rrbracket^{\mathrm{eff}}=\sqrt{{\overline{\llbracket v_{t} \rrbracket^{2}}}^{\Gamma}} .
$$

The results of Kreher (1990) have been extended to interfaces in Maalej et al. (2009), which yields

$$
\begin{aligned}
& \frac{1}{2} \frac{|\Gamma|}{|\Omega|}\left(\llbracket v_{n} \rrbracket^{\mathrm{eff}}\right)^{2}=\frac{\partial \Psi(\boldsymbol{D}, p, \varpi)}{\partial K_{n}} . \\
& \frac{1}{2} \frac{|\Gamma|}{|\Omega|}\left(\llbracket v_{t} \rrbracket^{\mathrm{eff}}\right)^{2}=\frac{\partial \Psi(\boldsymbol{D}, p, \varpi)}{\partial K_{t}} .
\end{aligned}
$$

\subsubsection{Mori Tanaka estimates}

The linear problem (20) will be solved using a generalized Mori-Tanaka scheme in order to take into account the matrix-inclusion morphology. In the case of spherical inclusions, it is well-known that the use of this scheme can be theoretically justified when the spatial distribution of the inclusions is isotropic. This assumption is adopted in the sequel. The radius of the spherical inclusions is $r_{0}$ and the volume fraction of the inclusionary phase is $\rho$. Accordingly, the specific surface of the interfaces is

$$
\frac{|\Gamma|}{|\Omega|}=\frac{3 \rho}{r_{0}} .
$$

Solution of problem (P1). The matrix-inclusion morphology suggests to resort to a Mori-Tanaka homogenization scheme in order to derive an estimate of $\mathbb{C}^{\text {hom }}$. However, the morphologically representative pattern is not a homogeneous inclusion but a composite inclusion made up of a rigid sphere surrounded by an elastic interface. The modified Eshelby problem of a sphere surrounded by a weak interface as introduced in Hashin (1991) has thus to be used. Transposing the results of Dormieux et al. (2010) derived for a self consistent scheme, the Mori-Tanaka estimate of the homogenized stiffness is $\mathbb{C}^{\text {hom }}=3 k^{\text {hom }} \mathbb{J}+2 \mu^{\text {hom }} \mathbb{K}$ where the compressibility and shear modulii are

$$
\begin{aligned}
& k^{\text {hom }}=\frac{1}{3} \frac{\left(3 k_{m}+4 \rho \mu_{m}\right) r_{0} K_{n}+(1-\rho) 12 k_{m} \mu_{m}}{(1-\rho) r_{0} K_{n}+4 \mu_{m}+3 \rho k_{m}}, \\
& 2 \mu^{\text {hom }}=\mu_{m} \frac{\left(\left((3+2 \rho) 4 \mu_{m}+(2+3 \rho) 3 k_{m}\right) r_{0} K_{n}+4 \mu_{m}\left((2+\rho) 8 \mu_{m}+(1+\rho) 9 k_{m}\right)\right) r_{0} K_{t}+\ldots}{\left(3(1-\rho)\left(k_{m}+2 \mu_{m}\right) r_{0} K_{n}+\left((4-3 \rho) 4 \mu_{m}+(3-2 \rho) 3 k_{m}\right) 2 \mu_{m}\right) r_{0} K_{t}+\ldots} \\
& \frac{\ldots\left(\left((3+\rho) 8 \mu_{m}+(5+3 \rho) 3 k_{m}\right) r_{0} K_{n}+(1-\rho)\left(8 \mu_{m}+9 k_{m}\right) 8 \mu_{m}\right) 2 \mu_{m}}{\ldots\left(3\left((5-2 \rho) k_{m}+(2-\rho) 4 \mu_{m}\right) r_{0} K_{n}+\left((2+3 \rho) 4 \mu_{m}+(3+2 \rho) 3 k_{m}\right) 8 \mu_{m}\right) \mu_{m}} .
\end{aligned}
$$

In the following developments, the limit case $K_{n} / K_{t} \rightarrow \infty$ will be of particular interest as seen from (11) and (19). The homogenized modulii then read

$$
\begin{aligned}
k^{\text {hom }, 1} & =\frac{3 k_{m}+4 \rho \mu_{m}}{3(1-\rho)} \\
2 \mu^{\text {hom }, 1} & =\mu_{m} \frac{\left(3 k_{m}(2+3 \rho)+4 \mu_{m}(2 \rho+3)\right) r_{0} K_{t}+2 \mu_{m}\left(8(\rho+3) \mu_{m}+3(3 \rho+5) k_{m}\right)}{3\left((1-\rho)\left(k_{m}+2 \mu_{m}\right) r_{0} K_{t}+\mu_{m}\left((5-2 \rho) k_{m}+4(2-\rho) \mu_{m}\right)\right)} .
\end{aligned}
$$


In view of the derivation of the potential energy (see (24)), it is useful to determine the matrix volume change $v_{1_{m}}$ as a function of the macroscopic strain rate $\boldsymbol{D}$. Solving of (P1) using a Mori-Tanaka scheme leads to

$$
v_{1_{m}}=b_{m} \mathbf{1}: \boldsymbol{D}
$$

where the coefficient $b_{m}$ is equal to

$$
b_{m}=(1-\rho) \frac{r_{0} K_{n}+4 \mu_{m}}{(1-\rho) r_{0} K_{n}+4 \mu_{m}+3 \rho k_{m}} .
$$

Solution of problem (P2). At the microscopic scale, the stress field linearly depends on $p+\varpi$. Hence, there is a second order tensor $\boldsymbol{B}_{2}$ such that $\boldsymbol{\sigma}_{2}=-\boldsymbol{B}_{2}(p+\varpi)$. The macroscopic stress is then $\boldsymbol{\Sigma}_{2}=-\overline{\boldsymbol{B}}_{2} \Omega(p+\varpi)$.

In the line of reasoning of the Maxwell-Betti theorem and its application to poroelasticity (Dormieux et al., 2002), first note that:

$$
|\Omega| \overline{\boldsymbol{d}_{1}: \mathbb{C}: \boldsymbol{d}_{2}}{ }^{\Omega}+|\Gamma| \overline{\llbracket \boldsymbol{v}_{1} \rrbracket \cdot \boldsymbol{K} \cdot \llbracket \boldsymbol{v}_{2} \rrbracket}{ }^{\Gamma}=|\Omega|{\overline{\boldsymbol{d}_{2}: \mathbb{C}: \boldsymbol{d}_{1}}}^{\Omega}+|\Gamma| \overline{\llbracket \boldsymbol{v}_{2} \rrbracket \cdot \boldsymbol{K} \cdot \llbracket \boldsymbol{v}_{1} \rrbracket}{ }^{\Gamma} .
$$

where the symmetry of $\mathbb{C}$ and $\boldsymbol{K}$ was used. Careful application of Hill's lemma (in its extended form to interfaces (Maalej et al., 2009)) to the crossed couples $\left(\boldsymbol{d}_{1}, \boldsymbol{\sigma}_{2}\right)$ and $\left(\boldsymbol{d}_{2}, \boldsymbol{\sigma}_{1}\right)$, with account for the prestress $p+\varpi$ then yields:

$$
{\overline{\boldsymbol{d}_{1}}}^{\Omega}:{\overline{\boldsymbol{\sigma}_{2}}}^{\Omega}+(p+\varpi) \mathbf{1}:(1-\rho){\overline{\boldsymbol{d}_{1}}}^{m}={\overline{\boldsymbol{d}_{2}}}^{\Omega}:{\overline{\boldsymbol{\sigma}_{1}}}^{\Omega} .
$$

Next, recalling the boundary conditions in (P1) and (P2) as well as (30), one obtains:

$$
-(p+\varpi){\overline{\boldsymbol{B}_{2}}}^{\Omega}: \boldsymbol{D}+(p+\varpi) b_{m} \mathbf{1}: \boldsymbol{D}=0 .
$$

The macroscopic stress of (P2) is finally derived as

$$
\boldsymbol{\Sigma}_{2}=-(p+\varpi) b_{m} \mathbf{1}
$$

In turn, let us determine the normalized matrix volume change $v_{2_{m}}$ which is involved in (24). To do so, the previous expression of the macroscopic stress in (P2) is confronted to the stress average rule (see Eqs. (35) to (38) for details)

$$
\boldsymbol{\Sigma}_{2}=-(p+\varpi) b_{m} \mathbf{1}=(1-\rho)\left(\mathbb{C}^{m}:{\overline{\boldsymbol{d}_{2}}}^{\Omega_{m}}-(p+\varpi) \mathbf{1}\right)+\frac{|\Gamma|}{|\Omega|} r_{0} \overline{\boldsymbol{n} \otimes \boldsymbol{K} \cdot \llbracket \boldsymbol{v}_{2} \rrbracket}{ }^{\Gamma},
$$

Applying the trace operator, the above equation simplifies into

$$
-3(p+\varpi) b_{m}=3 k_{m} v_{2_{m}}-(1-\rho) 3(p+\varpi)+\frac{|\Gamma|}{|\Omega|} r_{0} K_{n} \overline{\llbracket v_{2_{n}} \rrbracket}{ }^{\Gamma} .
$$

Since the macroscopic strain rate is null in problem (P2) and recalling that the inclusions are rigid (no strain), the strain average rule reduces to:

$$
0=v_{2_{m}}+\frac{|\Gamma|}{|\Omega|} \overline{\llbracket v_{2_{n}} \rrbracket} \Gamma .
$$

Finally, the matrix volume change depends on the prestress $-(p+\varpi)$ as

$$
v_{2_{m}}=-\frac{3\left(b_{m}-1+\rho\right)}{3 k_{m}-r_{0} K_{n}}(p+\varpi)=\frac{1}{N_{m}}(p+\varpi),
$$

where the modulus $N_{m}$ takes the form

$$
N_{m}=\frac{(1-\rho) r_{0} K_{n}+4 \mu_{m}+3 \rho k_{m}}{3 \rho(1-\rho)} .
$$


Complete linear problem. Adapting from Dormieux et al. (2006), the two macroscopic state equations of the complete linear problem are obtained by superposition of the three loading modes

$$
\begin{aligned}
\boldsymbol{\Sigma} & =\mathbb{C}^{\text {hom }}: \boldsymbol{D}-(p+\varpi) b_{m} \mathbf{1}+\varpi \mathbf{1}, \\
v_{m} & =b_{m} \mathbf{1}: \boldsymbol{D}+\frac{p+\varpi}{N_{m}}
\end{aligned}
$$

where $\mathbb{C}^{\text {hom }}$ is defined by $(28), b_{m}$ by $(31)$ and $N_{m}$ by $(32)$. (24)

The potential energy of the complete linear viscous problem is finally obtained by injection of (33) in

$$
\Psi(\boldsymbol{D}, p, \varpi)=\frac{1}{2} \boldsymbol{D}: \mathbb{C}^{\text {hom }}: \boldsymbol{D}-(p+\varpi) b_{m} \operatorname{tr} \boldsymbol{D}-\frac{(p+\varpi)^{2}}{2 N_{m}}+\varpi \operatorname{tr} \boldsymbol{D} .
$$

For forthcoming use, we also provide an alternative micro-to-macro expression of the macroscopic mean stress $\Sigma_{m}$. The starting point is the stress average rule $\Sigma_{m}=\overline{\sigma_{m}}$ which in turn reads:

$$
\Sigma_{m}=(1-\rho){\overline{\sigma_{m}}}^{m}+\rho{\overline{\sigma_{m}}}^{i}
$$

We now evaluate the average ${\overline{\sigma_{m}}}^{i}$ from the average ${\overline{T_{n}}}^{\Gamma}$ of the normal component. Indeed, considering a given particle $\mathcal{P} \subset \Omega_{i}$, one obtains:

$$
\frac{1}{|\mathcal{P}|} \int_{\mathcal{P}} \boldsymbol{\sigma} d V=\frac{1}{|\mathcal{P}|} \int \boldsymbol{z} \otimes \boldsymbol{T} d S .
$$

We now consider the case of a spherical particle with radius $r_{0}$. In this case, we note that $\boldsymbol{z}$ is $r_{0} \boldsymbol{n}$, where $\boldsymbol{n}$ is the unit normal vector to $\partial \mathcal{P}$. From the above equation, it is readily seen that:

$$
{\overline{\sigma_{m}}}^{\mathcal{P}}={\overline{T_{n}}}^{\partial \mathcal{P}}
$$

which eventually yields

$$
{\overline{\sigma_{m}}}^{i}={\overline{T_{n}}}^{\Gamma} .
$$

Introducing this result into (35) and recalling (20), we eventually obtain the following expression for $\Sigma_{m}$ :

$$
\Sigma_{m}=k_{m} v_{m}-(1-\rho) p+\rho\left(K_{n}{\overline{\llbracket v_{n} \rrbracket}}^{\Gamma}+\varpi\right) .
$$

\subsection{Non linear homogenization}

We now consider the non linear problem (2). It is formally identical to the linear one that has just been dealt with, up to the fact that the non linear modulii (5) are substituted for $\mathbb{C}_{m}$ and (11) or (18) for $\boldsymbol{K}$ in (20).

To account for the non linearity of the matrix stiffness tensor in a simplified manner, the idea is to assume that the non linear stiffness tensors can be viewed as functions of appropriate averages of the strain rate, referred to as "effective strain" (rate). In the modified secant method (Suquet, 1995, 1997), these effective estimates of the volume and deviatoric strain rates are quadratic averages over the matrix phase of these quantities. Namely, the effective values of the secant modulii (5) in the matrix phase are estimated using (25) as

$$
k_{m}^{\mathrm{eff}} \approx k_{m}\left(d_{v}^{\mathrm{eff}}, d_{d}^{\mathrm{eff}}\right) \quad ; \quad \mu_{m}^{\mathrm{eff}} \approx \mu_{m}\left(d_{v}^{\mathrm{eff}}, d_{d}^{\mathrm{eff}}\right) .
$$

Similarly, the non linearity of the interface stiffness is taken into account through the estimates of the jump of velocity components (26) across the interface

$$
K_{n}^{\mathrm{eff}} \approx K_{n}\left(\llbracket v_{n} \rrbracket^{\mathrm{eff}}, \llbracket v_{t} \rrbracket^{\mathrm{eff}}\right) \quad ; \quad K_{t}^{\mathrm{eff}} \approx K_{t}\left(\llbracket v_{n} \rrbracket^{\mathrm{eff}}, \llbracket v_{t} \rrbracket^{\mathrm{eff}}\right) .
$$




\section{Macroscopic criteria}

\subsection{Perfect interfaces}

To start with, perfect interfaces are considered. In this case, no jump of velocity is allowed at the interface, while the tangential and normal stiffnesses of the interface are infinite. Thus, problem (2) is solved based on the results of the linear problem (20) in the limit case where $K_{n}$ and $K_{t}$ are infinite with no prestress in the interfaces $(\varpi=0)$.

As far as the underlying linear problem is concerned, the previous decomposition in the three subproblems becomes trivial.

For (P1), the linear homogenized modulii (28) reduce to the classical Mori-Tanaka estimates for a matrix composite with perfectly bounded and infinitely stiff inclusions

$$
\begin{aligned}
k^{\mathrm{hom}} & =\frac{3 k_{m}+4 \rho \mu_{m}}{3(1-\rho)}, \\
2 \mu^{\mathrm{hom}} & =\mu_{m} \frac{3 k_{m}(2+3 \rho)+4 \mu_{m}(2 \rho+3)}{3(1-\rho)\left(k_{m}+2 \mu_{m}\right)} .
\end{aligned}
$$

For (P2), it is readily seen that the solution is $\left(\boldsymbol{v}_{2}=\mathbf{0}, \boldsymbol{\sigma}_{2}=-p \mathbf{1}\right)$ while the solution to (P3) is $\left(\boldsymbol{v}_{3}=\right.$ $\mathbf{0}, \boldsymbol{\sigma}_{3}=0$ ) (since $\varpi=0$ ).

Computations are carried out by substituting the homogenized modulii (42) in the potential energy with $p=c$ (see (5)) and $\varpi=0$. Then estimates of the strain rate are derived using (25) and the non linear equations (40) are solved for $d_{v}^{\text {eff }}$ and $d_{d}^{\text {eff }}$. The solution is reported in $\mu_{m}^{\text {eff }}$ :

$$
2 \mu_{m}^{\mathrm{eff}}=\frac{(1-\rho) b}{D_{d}} \sqrt{\frac{6(a+b)}{2(a+b)(2 \rho b+3 a) \beta^{2}+b q}},
$$

where the constant $q$ is defined at (48) and $\beta$ is the strain triaxiality ratio defined as

$$
\beta=D_{v} / D_{d} .
$$

Then, recalling (5), one obtains $k_{m}^{\text {eff }}=2 \mu_{m}^{\text {eff }} a / b$. Using these expressions of the effective matrix modulii in (42), the macroscopic stress $\Sigma$ is eventually obtained from the first equation of (33) which provides parametric equations for $\Sigma_{m}$ and $\Sigma_{d}$ as functions of $\beta$. Eliminating $\beta$ yields the equation of the boundary $\partial G^{\text {hom }}$, that is, the macroscopic criterion. As expected, the latter is isotropic. $\partial G^{\text {hom }}$ is an ellipse in the $\left(\Sigma_{m}, \Sigma_{d}\right)$ plane. The domain $G^{\text {hom }}$ is defined as:

$$
\begin{gathered}
\frac{\left(\Sigma_{m}+c\right)^{2}}{A}+\frac{\Sigma_{d}^{2}}{B}-1 \leqslant 0 \\
\text { where } A=a\left(1+\rho \frac{2 b}{3 a}\right) \quad ; \quad B=b\left(1+\rho \frac{9 a+4 b}{6(a+b)}\right) .
\end{gathered}
$$

It is readily seen that $G^{\text {hom }}$ contains the matrix criterion (see (3)). This result had already been obtained by Shen et al. (2012) using the same homogenization techniques.

\subsection{Tresca interfaces}

Next, the case where the interfaces are governed by a Tresca criterion (6) is considered. First, the macroscopic criterion is derived under the assumption of interface activation. Then the conditions for interface activation are derived. 


\subsubsection{Derivation of the macroscopic criterion when the interfaces are activated}

As one can see from (5) and (10), the considered underlying linear problem has a prestress $p=c$ in the matrix, no prestress in the interface $(\varpi=0)$, and an infinite normal modulus $K_{n}$ in the interface.

The effective value of the tangential jump of velocity $\llbracket v_{t} \rrbracket^{\text {eff }}$ across the interface is expressed as a function of the matrix effective modulii by use of (26) and (34) where $\mathbb{C}^{\text {hom }}$ is characterized by (29) and $K_{t}$ by (11). Doing so, a second order polynomial equation in $\llbracket v_{t} \rrbracket^{\text {eff }}$ appears. By definition, $\llbracket v_{t} \rrbracket^{\text {eff }}$ is positive, and the equation has only one or no positive root depending on the tangential strength $k$ of the interfaces. Two cases have to be studied

1. Inactive interfaces: when no real positive solution of the equation is available, the interfaces are not active, meaning that the yield condition of the interface criterion is not reached. In this case, $\llbracket v_{t} \rrbracket^{\text {eff }}=0$ and $K_{t} \rightarrow \infty$ so that the macroscopic criterion for perfect interfaces (45) is retrieved.

2. Active interfaces: the equation has a positive root $\llbracket v_{t} \rrbracket^{\text {eff }}>0$, which means that the interfaces are active.

The mathematical condition for interface activation will be provided later. The tangential velocity jump $\llbracket v_{t} \rrbracket^{\text {eff }}$, as well as $K_{t}^{\text {eff }}$ through (11) are now functions of the effective volume and deviatoric strain rates in the matrix. In turn, these matrix effective strain rates $d_{v}^{\text {eff }}$ and $d_{d}^{\text {eff }}$ are expressed by use of (25), (34) and (29) in which $k_{m}$ is substituted for $k_{m}=2 \mu_{m} a / b$ using (5). The expressions of $d_{v}^{\text {eff }}$ and $d_{d}^{\text {eff }}$ are then injected in $\mu_{m}$, resulting in a second order polynomial equation in $\mu_{m}^{\text {eff }}$ which has a single positive root. Explicit effective values of $k_{m}^{\text {eff }}$ and $K_{t}^{\text {eff }}$ are deduced, and substituted in $\mu^{\text {hom }}$ and $k^{\text {hom }}$ to find a parametric equation of the macroscopic stress states lying on the boundary of the macroscopic strength criterion using (33). The macroscopic stress state obtained from (33) lies on a portion of ellipse of equation

$$
\frac{\left(\Sigma_{m}+c\right)^{2}}{A}+\frac{\left(\Sigma_{d}-\Sigma_{d 0}\right)^{2}}{B} \leqslant 1
$$

If the interfaces are not active, the ellipses parameters are defined by (45) and $\Sigma_{d 0}=0$. Otherwise, when interfaces mechanisms are active, the parameters of the ellipse are

$$
\begin{aligned}
A & =a\left(1+\frac{2 \rho b}{3 a}\right)\left(1-\left(\frac{k}{k_{e}}\right)^{2}\right) \\
B & =\frac{(1-\rho) r b^{2}}{18 \rho(b+a) k_{e}^{2}}\left(1-\left(\frac{k}{k_{e}}\right)^{2}\right) \\
\Sigma_{d 0} & =\frac{\sqrt{5}(2 b+3 a)}{6(b+a)} \frac{b}{k_{e}^{2}} k
\end{aligned}
$$

where the following useful notations are introduced:

$$
\begin{aligned}
& t=(4-2 \rho) b+(5-2 \rho) a, \\
& q=(6+4 \rho) b+(6+9 \rho) a, \\
& r=(4 \rho+12) b+(9 \rho+15) a,
\end{aligned}
$$

and $k_{e}$ is a specific value of the tangential strength defined in (49).

\subsubsection{Conditions for interface activation}

At this stage, the condition for the activation of the interfaces has to be explicited. This condition is shown to depend on the macroscopic strain triaxiality ratio $\beta=D_{v} / D_{d}$. First, the existence of roots to the equation on $\llbracket v_{t} \rrbracket^{\text {eff }}$ imposes

$$
k^{2} \leqslant k_{e}^{2}=\frac{t b}{6 \rho(b+a)} .
$$


Further, one of the roots is positive if

$$
k^{2} \leqslant k_{c}^{2}(\beta)=\frac{5}{6} \frac{(b(2 b+3 a))^{2}}{2(b+a)^{2}(3 a+2 b \rho) \beta^{2}+(b+a) b q},
$$

where the inequality $k_{c}^{2}(\beta)<k_{e}^{2}$ is met irrespective of the value of $\beta$. Also note that $k_{c}(\beta)$ is an even decreasing function of $\beta$, thus reaching its maximum for $\beta=0$ (limit case of a purely deviatoric macroscopic strain rate).

To summarize, two cases have to be considered

- If $k>k_{c}(0)$, the interfaces are never active because the interfaces are too strong (Fig. 2a,2b): the macroscopic criterion is equal to the criterion with perfect interfaces (45).

- If $k \leqslant k_{c}(0)$, a critical value of the strain triaxiality ratio $\beta=D_{v} / D_{d}$ appears

$$
\beta_{c}^{2}=\frac{5}{12(3 a+2 b \rho)}\left(\frac{(2 b+3 a) b}{(b+a) k}\right)^{2}\left(1-\left(\frac{k}{k_{c}(0)}\right)^{2}\right) .
$$

The discussion concerning the status of the interfaces is controlled by the strain triaxiality ratio (44):

- if $\beta^{2} \geqslant \beta_{c}^{2}$, the interfaces are not active.

- if $\beta^{2}<\beta_{c}^{2}$, the interfaces are active. The effective matrix shear modulus is

$$
2 \mu_{m}^{\mathrm{eff}}=\frac{(1-\rho) b}{D_{d}} \sqrt{\frac{3 t}{\left(t(3 a+2 b \rho) \beta^{2}+(1-\rho) b r\right)}\left(1-\left(\frac{k}{k_{e}}\right)^{2}\right)}
$$

and the tangential velocity jump is

$$
\llbracket v_{t} \rrbracket^{\mathrm{eff}}=\frac{\sqrt{5}}{3} \frac{(2 b+3 a) r_{0} D_{d}}{t}\left(1-\frac{k}{k_{c}(\beta)}\right) .
$$

Hence, provided that the tangential strength of the interface is sufficiently low $\left(k \leqslant k_{c}(0)\right)$, the boundary $\partial G^{\text {hom }}$ is a combination of two ellipses : for high triaxiality ratios $\left(\beta^{2} \geqslant \beta_{c}^{2}\right)$, the criterion for perfect interfaces is retrieved while for low triaxiality ratios $\left(\beta^{2} \leqslant \beta_{c}^{2}\right)$, the criterion is weakened by the activation of the interfaces and branches into a smaller ellipse (Fig. 2c,2d), which center lies on the vertical axis $\Sigma_{m}=-c$. Note that the two ellipses are tangent at their intersections for $\beta^{2}=\beta_{c}^{2}$. At fixed matrix strength properties and inclusions concentration, reduction of the interface tangential strength $k$ results in a lower deviatoric strength, whereas the hydrostatic strengths, either in compression or traction, are unchanged.

\subsection{Mohr-Coulomb interfaces}

Finally, the case where the interfaces strength is governed by a Mohr-Coulomb criterion (12) is considered. The underlying linear problem makes full use of the results derived in section 3.1, with prestress in the matrix $(p=c)$ and in the interfaces $\left(\varpi=T_{p}\right)$.

\subsubsection{Derivation of the macroscopic criterion when the interfaces are activated}

Proceeding as for the Tresca interfaces case, the effective tangential jump of velocity $\llbracket v_{t} \rrbracket^{\text {eff }}$ across the interface is expressed as a function of the matrix effective modulii and the interface function $\mathcal{F}$ by use of (26) and (34) where $\mathbb{C}^{\text {hom }}$ is characterized by (29) and $K_{t}$ by (18). Recalling from (5), $k_{m}=2 \mu_{m} a / b$, and the only conditionally positive root is

$$
\llbracket v_{t} \rrbracket^{\mathrm{eff}}=\frac{6(1-\rho)(a+b) \alpha \mathcal{F}+2 \sqrt{5} \mu^{\mathrm{eff}}(2 b+3 a) D_{d}}{6 t \mu^{\mathrm{eff}}} r_{0} .
$$




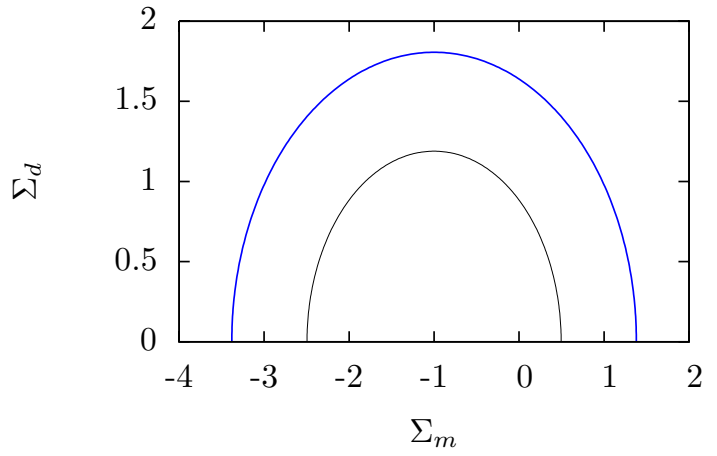

(a) $k>k_{e}$

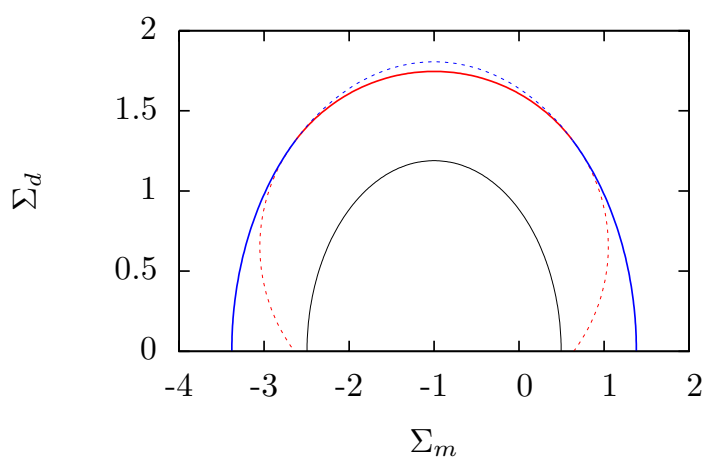

(c) $k=0.8<k_{c}(0)$

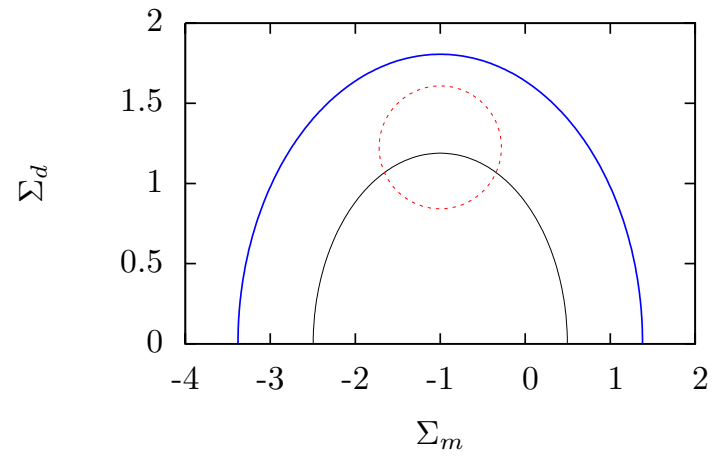

(b) $k_{c}(0)<k=1.5<k_{e}$

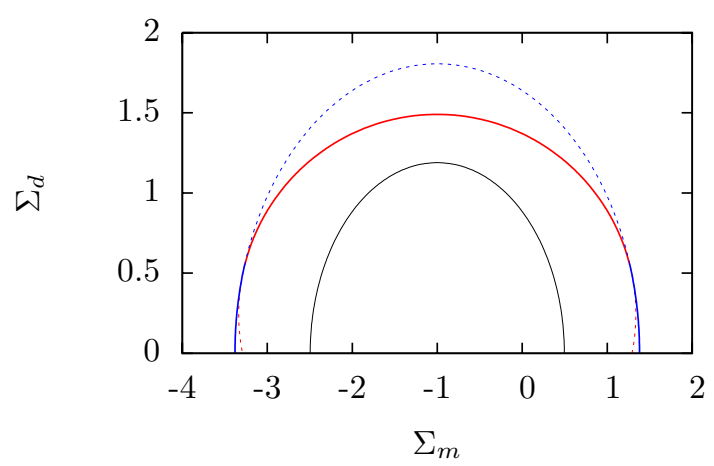

(d) $k=0.3<k_{c}(0)$

Figure 2: Macroscopic strength criterion for Tresca interfaces (thick blue-red line). The blue line corresponds to inactive interfaces and the red line to active interfaces, for different values of the interfaces tangential strength $k$. The fine solid black line is the matrix criterion for $a=5, b=2$ and $c=1$. The inclusion volume fraction is $\rho=0.5$. The dotted blue-red line are the remaining part of the ellipsis which are not part of the criterion boundary. The critical tangential strength values are $k_{e}=1.57$ and $k_{c}(0)=1.12$. 
The normal velocity jump can be determined either from the normality rule $\llbracket v_{n} \rrbracket^{\text {eff }}=\alpha \llbracket v_{t} \rrbracket^{\text {eff }}$ (see section 2.4) or consistently with the quadratic estimate (26). Moreover, the direct estimate ${\overline{\llbracket v_{n} \rrbracket}}^{\Gamma}$ proves to be equal to the quadratic estimate $\llbracket v_{n} \rrbracket^{\text {eff }}$.

The interface parameter $\mathcal{F}(Y)$ (see (16)) is determined from the comparison of the macroscopic expression of the mean macroscopic stress derived from (33):

$$
\Sigma_{m}=k^{\mathrm{hom}} D_{v}-c b_{m}+\left(1-b_{m}\right) T_{p}
$$

and the direct average of the microscopic mean stress derived from (39):

$$
\Sigma_{m}=k_{m} v_{m}-(1-\rho) c+\rho\left(K_{n} \llbracket v_{n} \rrbracket^{\text {eff }}+T_{p}\right)
$$

where we have used the fact that $\llbracket v_{n} \rrbracket^{\text {eff }}={\overline{\llbracket v_{n} \rrbracket}}^{\Gamma}$. The matrix volumic strain rate $v_{m}$ is expressed thanks to the second macroscopic state equation (33). Then, according to the interface state equation $(16), K_{n} \llbracket v_{n} \rrbracket^{\text {eff }}+$ $T_{p}=h+\mathcal{F}$. Furthermore, in the limit case $K_{n}, T_{p} \rightarrow \infty$, the interface fictitious behavior is such that

$$
b_{m} \rightarrow 1 \quad ; \quad\left(1-b_{m}\right) T_{p} \rightarrow-\frac{\rho\left(3 k_{m}+4 \mu_{m}\right)}{(1-\rho) r_{0}} \llbracket v_{n} \rrbracket^{\mathrm{eff}} \quad ; \quad \frac{T_{p}}{N_{m}} \rightarrow-\frac{3 \rho}{r_{0}} \llbracket v_{n} \rrbracket^{\mathrm{eff}}
$$

From the comparison of the two above expressions of the macroscopic mean stress, we get:

$$
\mathcal{F}=\frac{3 k^{\mathrm{eff}}+4 \mu^{\mathrm{eff}}}{3(1-\rho)} D_{v}-\frac{3 \rho k^{\mathrm{eff}}+4 \mu^{\mathrm{eff}}}{r_{0}(1-\rho)} \llbracket v_{n} \rrbracket^{\mathrm{eff}}-c-h
$$

The last step proceeds as follows: An equation on $\llbracket v_{t} \rrbracket^{\text {eff }}$ is to be determined from substitution of $(55)$ into (54) with the normality rule $\llbracket v_{n} \rrbracket^{\text {eff }}=\alpha \llbracket v_{t} \rrbracket^{\text {eff }}$ and the identity $k^{\text {eff }}=2 \mu^{\text {eff }} a / b$. Akin to the Tresca interfaces, two cases have to be studied while solving this equation to get $\llbracket v_{t} \rrbracket^{\text {eff }}$ as a function of $\mu^{\text {eff }}, D_{v}$ and $D_{d}$ :

1. Inactive interfaces: when this equation has no positive solution, this physically means that the interfaces are not active, so that $\llbracket v_{t} \rrbracket^{\text {eff }}=0$, thus $K_{t} \rightarrow \infty$ and the macroscopic criterion for perfect interfaces (45) is retrieved.

2. Active interfaces: the equation has a positive root $\llbracket v_{t} \rrbracket^{\text {eff }}>0$, which means the interfaces are active.

In the case where interfaces are active, the effective matrix modulii have to be determined assuming a strictly positive value of $\llbracket v_{t} \rrbracket^{\text {eff }}$ exists. For the sake of clarity, the following additional notations are introduced:

$$
\begin{aligned}
u & =2(a+b)(3 \rho a+2 b) \alpha^{2}+b t, \\
w & =2(a+b) q \alpha^{2}+5 b(3 a+2 b \rho) .
\end{aligned}
$$

Assuming $\llbracket v_{t} \rrbracket^{\text {eff }}>0$, the effective value $\llbracket v_{n} \rrbracket^{\text {eff }}$ is deduced from the normality rule and $K_{t}^{\text {eff }}$ from (18). Then, the matrix strain rate effective values are expressed using (25). Since the interfaces normality rule is asymptotically met, the expression is taken in the limiting case (19) as for the calculation of $\mathcal{F}$. The effective strain rate estimates are cast in (5) to get a second order equation in $\mu_{m}^{\text {eff }}$, which has a positive root in the case where $(h+c)^{2} \leqslant h_{4}^{2}$ with

$$
h_{4}^{2}=\frac{u}{6 \rho(a+b) \alpha^{2}}
$$

The effective matrix shear modulus $\mu_{m}^{\text {eff }}$ finally is

$$
2 \mu_{m}^{\mathrm{eff}}=(1-\rho) \sqrt{\frac{3 b u}{A^{\prime} D_{v}^{2}+B^{\prime} D_{d}^{2}-2 C^{\prime} D_{v} D_{d}}\left(1-\left(\frac{h+c}{h_{4}}\right)^{2}\right)},
$$


where the following notations have been introduced

$$
\begin{aligned}
& A^{\prime}=12 a(a+b)(1-\rho)^{2} \alpha^{2}+(3 a+2 b \rho) t, \\
& B^{\prime}=(3 \rho a+2 b) q \alpha^{2}+b(1-\rho) r, \\
& C^{\prime}=\sqrt{5} \rho(2 b+3 a)^{2} \alpha .
\end{aligned}
$$

The tangential velocity jump, whose sign still has to be discussed, is

$$
\llbracket v_{t} \rrbracket^{\mathrm{eff}}=\frac{r_{0}}{3 u}\left((2 b+3 a)\left(\sqrt{5} b D_{d}+2 \alpha(a+b) D_{v}\right)-\frac{3(1-\rho) b(a+b) \alpha(h+c)}{\mu_{m}^{\mathrm{eff}}}\right) .
$$

The effective modulus $k_{m}^{\text {eff }}$ is derived from $\mu_{m}^{\text {eff }}(58)$ by $(5)$ while $K_{t}^{\text {eff }}$ (see $(18)$ ) is obtained from $\llbracket v_{t} \rrbracket^{\text {eff }}$ $(60)$ and $\mathcal{F}(55)$. These quantities are finally introduced into (29) in order to get $\mu^{\text {hom }}$ and $k^{\text {hom }}$. The macroscopic stress states $\left(\Sigma_{m}, \Sigma_{d}\right)$ at the boundary of the macroscopic strength criterion are retrieved from (33). The associated support function for the active interface part of the strength criterion is recognized to be

$$
\pi_{\mathrm{act}}^{\mathrm{hom}}(\boldsymbol{D})=\sqrt{\frac{3 b}{u}\left(1-\left(\frac{h+c}{h_{4}}\right)^{2}\right)\left(A^{\prime} D_{v}^{2}+B^{\prime} D_{d}^{2}-2 C^{\prime} D_{v} D_{d}\right)}+\Sigma_{m 0} D_{v}+\Sigma_{d 0} D_{d}
$$

with

$$
\Sigma_{m 0}=\frac{(h+c) \alpha(2 b+3 a) \rho}{u} 2(a+b) \alpha-c \quad ; \quad \Sigma_{d 0}=\frac{(h+c) \alpha(2 b+3 a) \rho}{u} \sqrt{5} b .
$$

The active interface part of the macroscopic strength criterion $f_{\text {act }}^{\text {hom }}$, of which $\pi_{\text {act }}^{\text {hom }}(61)$ is the support function, has for boundary a slant ellipse in the $\left(\Sigma_{m}, \Sigma_{p}\right)$ plane, centered at $\left(\Sigma_{m 0}, \Sigma_{p 0}\right)$ and characterized by the equation

$$
f_{\mathrm{act}}^{\mathrm{hom}}(\boldsymbol{\Sigma})=\frac{\left(\Sigma_{m}-\Sigma_{m 0}\right)^{2}}{A}+\frac{\left(\Sigma_{d}-\Sigma_{d 0}\right)^{2}}{B}+\frac{2\left(\Sigma_{m}-\Sigma_{m 0}\right)\left(\Sigma_{d}-\Sigma_{d 0}\right)}{C}-1,
$$

with the following ellipse parameters defined using (48), (59) and (57) as

$$
\begin{aligned}
& A=\frac{s}{B^{\prime}} \quad ; \quad B=\frac{s}{A^{\prime}} \quad ; \quad C=\frac{s}{C^{\prime}}, \\
& \text { with } s=\frac{(1-\rho) b}{3}\left(6 a(1-\rho) q \alpha^{2}+(3 a+2 b \rho) r\right)\left(1-\left(\frac{h+c}{h_{4}}\right)^{2}\right) .
\end{aligned}
$$

\subsubsection{Conditions for interface activation}

Let us now fully explicit the condition for interfaces activation: To do so, the sign of $\llbracket v_{t} \rrbracket^{\text {eff }}$ as a function of $h+c$ and $\beta=D_{v} / D_{d}$ has now to be studied and the cases of activation of the interfaces discussed.

The discussion is a bit technical and requires to introduce 4 parameters denoted by $h_{i}(i=1, \ldots, 4)$. $h_{4}^{2}$ was defined in (57) while the others parameters are:

$$
\begin{aligned}
& h_{1}^{2}=\frac{(2 b+3 a)^{2}}{3(3 a+2 b \rho)}, \\
& h_{2}^{2}=\frac{5 b(2 b+3 a)^{2}}{6(a+b) q \alpha^{2}}, \\
& h_{3}^{2}=\frac{w(2 b+3 a)^{2}}{6(b+a)(3 a+2 \rho b) q \alpha^{2}} .
\end{aligned}
$$


It can be shown that:

$$
h_{1}<h_{3}<h_{4} \quad ; \quad h_{2}<h_{3} .
$$

The sign of $h_{1}-h_{2}$ depends on the value of $\alpha$ as compared to

$$
\alpha_{c}^{2}=\frac{5 b(3 a+2 \rho b)}{2(a+b) q} .
$$

If $\alpha>\alpha_{c}$, then $h_{2}<h_{1}$, else $h_{2} \geqslant h_{1}$.

1. Activation of the interfaces is not possible for $(h+c)^{2}>h_{4}^{2}$ defined in (57), that is if their tensile strength is too high (Fig. 3a). The criterion for perfect interfaces is retrieved.

2. Activation is possible only under the additional condition $(h+c)^{2} \leqslant h_{3}^{2}$ which is a more stringent condition than the first one since $h_{3}<h_{4}$. When $h_{3}^{2}<(h+c)^{2}<h_{4}^{2}$, a positive root $\mu^{\text {eff }}$ exists but has no physical relevancy since the equation for $\llbracket v_{t} \rrbracket^{\text {eff }}$ has no positive root. More precisely, the macroscopic criterion based on (63) mathematically exists: it is strictly included in the ellipse defined by (45) (non active interfaces) and does not intersect with it. Nevertheless, it is not part of the boundary of the strength criterion (Fig. 3b) since $\llbracket v_{t} \rrbracket^{\text {eff }}<0$.

3. In the intermediate case $h_{1}<h+c<h_{3}$, interfaces are active if the strain triaxiality ratio (44) $\beta \in\left[\beta_{c-} ; \beta_{c+}\right]$ where

$$
\beta_{c \pm}=\frac{\sqrt{5} b(2 b+3 a) \pm(h+c) \sqrt{3 b w\left(1-\left((h+c) / h_{3}\right)^{2}\right)}}{2(a+b)(2 b+3 a) \alpha\left(\left((h+c) / h_{1}\right)^{2}-1\right)} .
$$

The intersection of the two parts of the macroscopic strength criterion occurs in the two points corresponding to $\beta_{c \pm}$ (Fig. 3c).

The sign of the critical value $\beta_{c-}$ is physically meaningful: When $\beta_{c-}>0$, the corresponding point on the macroscopic criterion lies on the right of the top of the ellipse. In this case, the maximum of the deviatoric stress is reached in a state where the interfaces are not activated. Interface activation is restricted to high values of the macroscopic mean stress. In contrast, if $\beta_{c-}<0$, the corresponding point on the macroscopic criterion lies on the left of the top of the ellipse which implies that the maximum of the deviatoric stress is reached in a state where the interfaces are indeed activated. This is consistent with the fact that $\beta_{c-}$ is an increasing function of $h$. Accordingly, the sign of $\beta_{c-}$ is determined as follows: $\beta_{c-}$ is negative when $(h+c)^{2}<h_{2}^{2}$ and positive otherwise. Note that $\beta_{c-}$ is not singular for $h+c=h_{1}$ since it simplifies into

$$
\beta_{c-}=\frac{5 b^{2}(2 b+3 a)\left(\left((h+c) / h_{2}\right)^{2}-1\right)}{2(a+b) \alpha\left(\sqrt{5} b(2 b+3 a)+(h+c) \sqrt{3 b w\left(1-\left((h+c) / h_{3}\right)^{2}\right)}\right)} .
$$

4. If $h+c<h_{1}, \beta_{c+} \rightarrow \infty$ and interfaces are always active if $\beta>\beta_{c-}$. The intersection of the two parts of the macroscopic strength criterion occurs at a single point (Fig. 3d). In this case, interfaces are active for a purely isotropic traction loading mode $(\beta \rightarrow \infty)$.

Thus, when the interfaces tensile strength is low enough $\left(h+c<h_{3}\right)$, the criterion (45) corresponding to perfect interfaces is truncated and symmetry along the plane $\Sigma_{m}=-c$ is broken. The ellipse for the active interfaces part of the strength criterion boundary (64) is tangent in $\beta_{c \pm}$ to the ellipse for non active interfaces (45), so the criterion has no angular point at the transition. At a given matrix strength and inclusion concentration, the reinforcement effect is seen to decrease with reduction of the interface tensile strength $h$ or friction angle $\alpha$ (Fig. 3). Finally, it is worth noting that the Tresca interfaces case is retrieved from the Mohr-Coulomb case in the limit $\alpha \rightarrow 0$ and $h \rightarrow \infty$ with $h=k / \alpha$. 


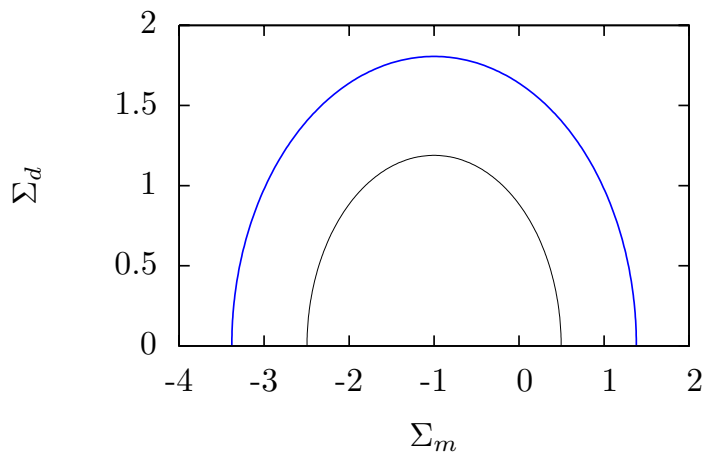

(a) $h+c>h_{4}$

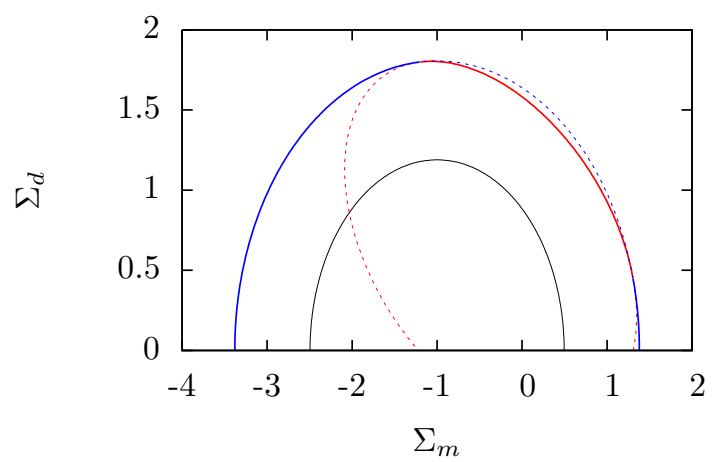

(c) $h_{1}<h+c=3.5<h_{3}$

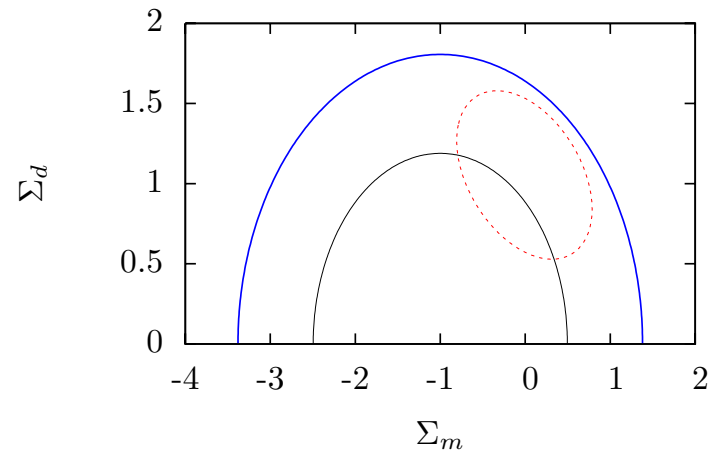

(b) $h_{3}<h+c=5.5<h_{4}$

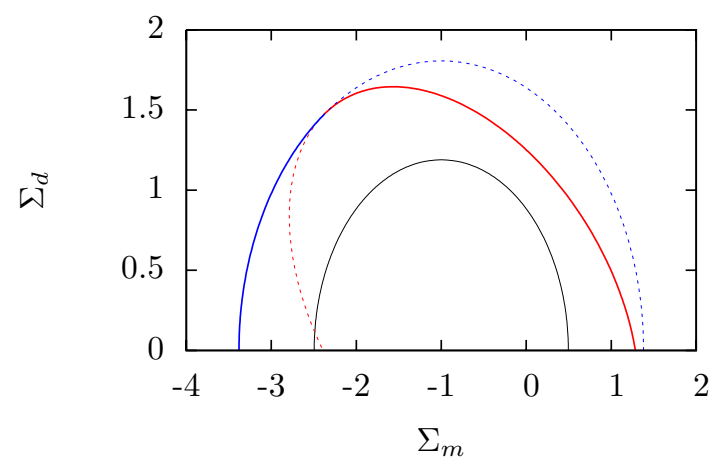

(d) $h+c=1.5<h_{1}$

Figure 3: Macroscopic strength criterion for Mohr-Coulomb interfaces (thick blue-red line). The blue line corresponds to inactive interfaces and the red line to active interfaces, for different values of the interfaces tensile strength $h$. The fine solid black line is the matrix criterion for $a=5, b=2$ and $c=1$. The inclusion volume fraction is $\rho=0.5$ and the friction angle $\alpha=0.3$. The dotted blue-red line are the remaining part of the ellipsis which do not belong to the criterion boundary. The critical interface strength values are $h_{1}=2.66, h_{2}=3.73, h_{3}=4.58$ and $h_{4}=5.93$. 
For the three cases of interface criteria under study - perfect, Tresca and Mohr-Coulomb - the homogenized strength criteria estimated by the modified secant modulii method appear to depend only on the first and second invariants of the macroscopic stress tensor. However, Tresca and Mohr-Coulomb criteria can generate a dependence to the third invariant of the macroscopic stress tensor. It would be interesting in future works to assess this effect by resorting to a numerical strength homogenization method.

\section{Conclusion}

In this paper, the effective strength of a matrix-inclusion composite has been considered. Specifically, the reinforcement of the matrix strength by rigid inclusions has been studied in the case where the matrix strength domain is delimited by an ellipse in the mean-deviatoric stress plane. The main focus of this work was the matrix-inclusion interface effects.

Three types of interfaces have been considered, characterized by a strength criterion on the stress vector acting upon the interface : perfect interfaces, Tresca interfaces for which the tangential stress vector is uniformly bounded and Mohr-Coulomb interfaces which model cohesive and frictional contact.

The effective strength has been derived within the continuum micromechanics framework. The boundary of the macroscopic strength criterion has been determined by identification of macroscopic stress states corresponding to a fictitious non linear viscous problem defined on a representative elementary volume. The non linear state equations for each constituent have been derived from the different kinds of strength criterion considered, which required a regularization of the interface criteria. The latter involve prestress terms which depend on the interface type at stake. This non linear problem has been solved by an implementation of the so-called modified secant method extended to the case of prestressed phases and interfaces. Estimates for the underlying prestressed linear problem have been provided using a Mori-Tanaka scheme based on a modified Eshelby problem in order to account for interface effects.

Closed form expressions of the effective strength have been proposed for the three interfaces types considered. First, in the case of perfect interfaces, a reinforcement is always observed. The effective criterion is an ellipse in the mean-deviatoric stress plane, concentric to the matrix criterion one, whose size varies linearly with inclusions concentration.

Second, in the case of Tresca interfaces, two situations have been observed. If the tangential strength of the interface is above a threshold which depends on microstructural properties, the interface mechanisms are not active, and the effective criterion is identical to the one for perfect interfaces. In turn, provided that the tangential strength of the Tresca interfaces is low enough, the effective criterion results from the truncation of the one for perfect interfaces by a parallel but smaller ellipse at deviatoric loads, for which interfacial mechanisms are active. The transition between the two parts of the effective criterion is controlled by the strain triaxiality ratio. This hybrid criterion is regular, without angles at the transition points between the two parts and symmetric.

Third, in the case of Mohr-Coulomb interfaces as compared to Tresca interfaces, the part of the effective criterion corresponding to the activation of the interfaces is located on an ellipse with slant axes. The local non symmetry of the Mohr-Coulomb criterion (which accounts for the sign of the normal stress) is transmitted to the macroscopic criterion: The latter has lost its symmetry with respect to the center of the matrix criterion. As expected, interface activation first occurs at higher values of the macroscopic mean stress.

The homogenization strategy adopted in this work is based on the modified secant modulii method. Hence, the homogenized criteria which have been derived are only estimates of the actual macroscopic criteria. To assess the herein presented criteria, numerical simulations should be carried out in future research on representative patterns such as a composite sphere made up of a rigid core surrounded by a matrix, with Tresca or Mohr-Coulomb interfaces between the two phases. We also expect that such simulations could allow to quantify the effect of the third invariant on the homogenized strength. 


\section{References}

Barthélémy, J.F., 2005. Approche micromécanique de la rupture et de la fissuration dans les géomatériaux. Ph.D. thesis. Ecole Nationale des Ponts et Chaussées.

Barthélémy, J.F., Dormieux, L., 2004. A micromechanical approach to the strength criterion of drucker-prager materials reinforced by rigid inclusions. International Journal for Numerical and Analytical Methods in Geomechanics 28, 565-582.

de Buhan, P., 1986. Approche fondamentale du Calcul à la Rupture des ouvrages en sols renforcés. Ph.D. thesis. Université Pierre et Marie Curie, Paris.

Dormieux, L., Jeannin, L., Bemer, E., Le, T.H., Sanahuja, J., 2010. Micromechanical models of the strength of a sandstone. International Journal for Numerical and Analytical Methods in Geomechanics 34, 249-271.

Dormieux, L., Kondo, D., Ulm, F.J., 2006. Microporomechanics. Wiley.

Dormieux, L., Molinari, A., Kondo, D., 2002. Micromechanical approach to the behavior of poroelastic materials. Journal of the Mechanics and Physics of Solids 50, 2203-2231.

Dormieux, L., Sanahuja, J., Maalej, Y., 2007. Résistance d'un polycristal avec interfaces intergranulaires imparfaites. Comptes Rendus de Mecanique 335, 25-31.

Greco, F., Leonetti, L., Lonetti, P., 2013. A two-scale failure analysis of composite materials in presence of fiber/matrix crack initiation and propagation. Composite Struct. 95, 582-597.

Gurson, A., 1977. Continuum theory of ductile rupture by void nucleation and growth. Part I: Yield criteria and flow rules for porous ductile media. J. Engng. Matl. Tech. Trans. ASME 99, 2-15.

Hashin, Z., 1991. The spherical inclusion with imperfect interface. Journal of Applied Mechanics 58, $444-449$.

He, Z., Dormieux, L., Lemarchand, E., Kondo, D., 2013. Cohesive mohr-coulomb interface effects on the strength criterion of materials with granular-based microstructure. European Journal of Mechanics A/Solids 42, 430-440.

Kreher, W., 1990. Residual stresses and stored elastic energy of composites and polycrystals. Journal of the Mechanics and Physics of Solids 38, 115-128.

Leblond, J.B., Perrin, G., Suquet, P., 1994. Exact results and approximate models for porous viscoplastic solids. Int. J. Plast. 10, 213-235.

Maalej, Y., Dormieux, L., Sanahuja, J., 2009. Micromechanical approach to the failure criterion of granular media. European Journal of Mechanics A/Solids 28, 647-653.

Maghous, S., Dormieux, L., Barthélémy, J.F., 2009. Micromechanical approach to the strength properties of frictional geomaterials. European Journal of Mechanics A/Solids 28, 179-188.

Mantič, V., 2009. Interface crack onset at a circular cylindrical inclusion under a remote transverse tension. Application of a coupled stress and energy criterion. Int. J. Sol. Struct. 46, 1287-1304.

Salencon, J., 1990. An introduction to the yield design theory and its applications to soil mechanics. European Journal of Mechanics 9, 477-500.

Sanahuja, J., Dormieux, L., 2005. Résistance d'un milieu poreux à phase solide hétérogène. Comptes Rendus de Mecanique $333,818-823$

Shen, W., Dormieux, L., Kondo, D., Shao, J., 2012. A closed-form three scale model for ductile rocks with a plastically compressible porous matrix. Mech. Mater. 59, 73-86.

Suquet, P., 1983. Analyse limite et homogénéisation. CRAS 296, 1355-1358.

Suquet, P., 1995. Overall properties of nonlinear composites: a modified secant moduli approach and its link with PonteCastaneda's nonlinear variational procedure. C. R. Acad. Sci. Paris 320, 563-571.

Suquet, P., 1997. Effective behavior of nonlinear composites, in: Continuum Micromechanics. Springer-Verlag. 